\title{
Progress in nuclear analyses of the ITER TBM Port Plug with Dummy TBMs
}

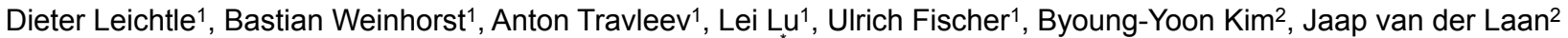 \\ ${ }^{1}$ Karlsruhe Institute of Technology, Hermann-von-Helmholtz-Platz 1, Eggenstein-Leopoldshafen, Germany \\ 2ITER Organization, Route de Vinon-sur-Verdon, CS 90 046, St. Paul Lez Durance Cedex, France
}

\section{Introduction}

- Test Program for Tritium Breeding Modules (TBM) in ITER equatorial ports

- TBM-sets (TBM + shield) to be replaced by Dummy-TBMs in case a TBM-set is not available.

- Maintenance within Port Interspace areas require hands-on operations.

- Nuclear analysis to compute Shutdown Dose Rates (SDDR) at $10^{6} \mathrm{~s}$ (ca. 12 days) after shutdown, with respect to $100 \mu \mathrm{Sv} / \mathrm{h}$ limit.

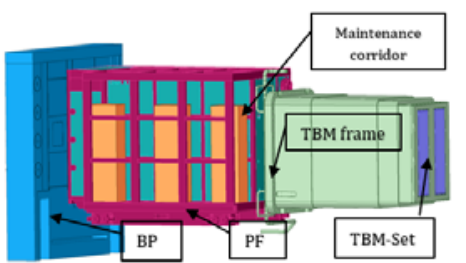

Main equipment of Test Blanket System, with Bioshield Plug (BP), Pipe Forest (PF), maintenance corridor and TBM Port Plug (Frame and TBM-Set or Dummy-TBM)

\section{Computational Methodology}

- R2S calculations of SDDR using MCNP6 and FISPACT-II, global model

- Superimposed Cartesian mesh of $3 \mathrm{~cm} / 15 \mathrm{~cm}$ spacing (in equatorial port) and $30 \mathrm{~cm}$ (in tokamak)

- Weight Window mesh for variance reduction by ADVANTG3 simulations.

- Operational scenarios:

- Short TBM relevant operation (first 4 years of nuclear operation)

- Full ITER SA2 operation (14 years of nuclear operation, $0.3 \mathrm{MWyr} / \mathrm{m}^{2}$ )

\section{SDDR calculations}

- Responses in human-body size tallies in maintenance corridor and as 3D radiation maps.

- Significant contribution, up to $\sim 85 \%$, by external structures, e.g. port duct walls.

- Minor contributions by Dummy-TBM ( 2 $\mu \mathrm{Sv} / \mathrm{h})$ and TBM-frame ( 20 $\mu \mathrm{Sv} / \mathrm{h})$.

- SDDR in PF corridor is above limit, also for short scenario, except in PF entry area.

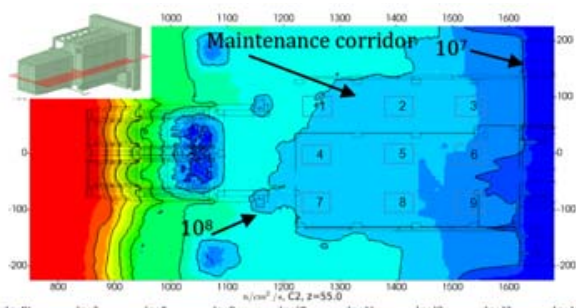

Neutron flux distribution across TBM port (C2)

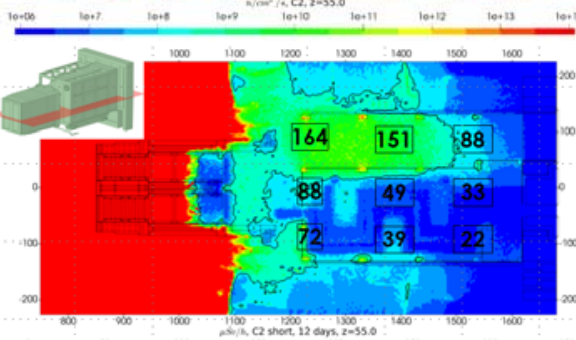

SDDR map (C2) with PF \#02 in PI, short irradiation

\section{Neutronics Models}

- Reference model of ITER tokamak sector, C-Model V1 R2.1.

- New MCNP model of TBM Port Plug, Dummy-TBM, Pipe Forest \#02 ( $\mathrm{HCCB}(\mathrm{CN})+\mathrm{LLCB}(\mathrm{IN})$ piping), and Bioshield Plug from CAD models.

- Simplification and conversion to MCNP geometry according to established ITER guidelines with high-level of details

- Configurations (for Equatorial Port \#02):

- C1: Empty Port-Interspace (+ BP with pipe-sections \& air gaps)

- C2: With Pipe Forest (connected to BP with pipe-sections \& air gaps)

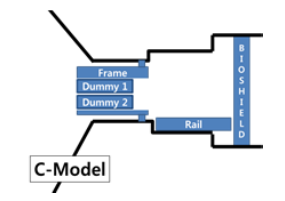

Configuration C1 schematic

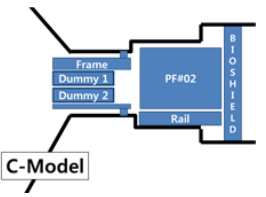

Configuration C2 schematic
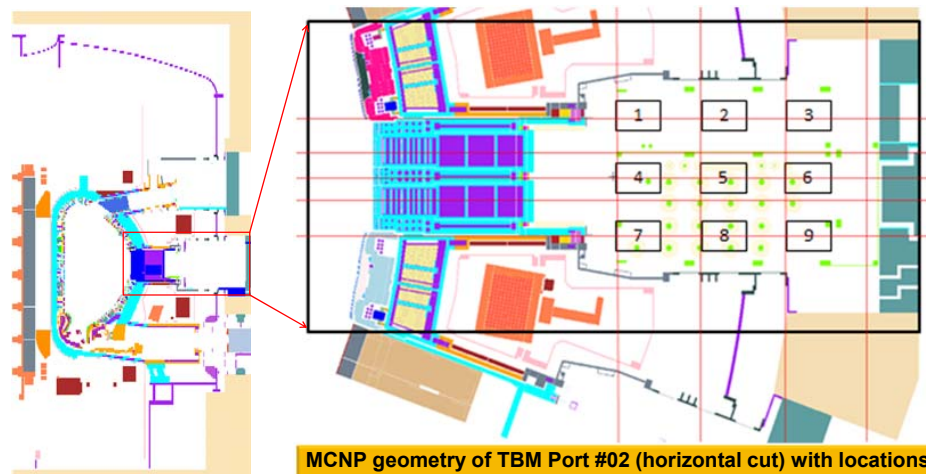

MCNP geometry of TBM Port \#02 (horizontal cut) with locations of human-body size tallies

\section{Conclusions}

- Models and analyses at pre-PDR maturity are provided for SDDR relevant for hands-on maintenance operations within Port Interspace of TBM Port

SDDR in PF entry area are compliant with ITER limit; in other areas higher by about a factor of 2 .

- Contribution of the TBM Port Plug is only up to $15 \%$ of total SDDR.

- Further reduction of SDDR by appropriate design choices and additional shielding structures is still required.

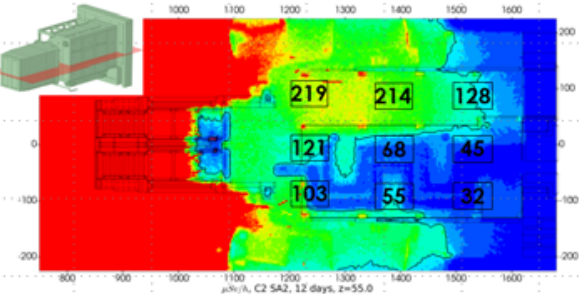

SDDR map (C2) with PF \#02 in PI, full ITER SA2 irradiation

\begin{tabular}{|c|c|c|c|}
\hline Part of model & Position 1 & Position 2 & Position 3 \\
\hline Equatorial port & 161 & 133 & 83 \\
\hline \multicolumn{4}{|l|}{ Components } \\
\hline 2 Dummy TBMs & 2 & 1 & 0.5 \\
\hline TBM frame & 20 & 8 & 4 \\
\hline Rest & 139 & 124 & 79 \\
\hline \begin{tabular}{|l|} 
External \\
structures
\end{tabular} & 30 & 41 & 16 \\
\hline Total & 191 & 174 & 99 \\
\hline
\end{tabular}

Contributions to SDDR $[\mu \mathrm{Sv} / \mathrm{h}]$ in empty Port Interspace (C1)
KIT - University of the State of Baden-Wuerttemberg and National Research Center of the Helmholtz Association $30^{\text {th }}$ Symposium on Fusion Technology, Giardini Naxos, Italy September 16-21, 2018
Acknowledgements and Disclaimer

This work was funded by the ITER Organization under contract IO/17/CT/4300001445. The views and opinions expressed herein do not necessarily reflect those of the ITER Organization. 